\title{
Entropic torque
}

\author{
R. Roth ${ }^{1,2}$, R. van Roij ${ }^{3}$, D. Andrienko ${ }^{4 *}$, K. R. Mecke ${ }^{1,2}$, and S. Dietrich ${ }^{1,2}$ \\ ${ }^{1}$ Max-Planck-Institut für Metallforschung, Heisenbergstrasse 1, D-70569 Stuttgart, Germany \\ ${ }^{2}$ Institut für Theoretische und Angewandte Physik, Universität Stuttgart, Pfaffenwaldring 57, D-70569 Stuttgart, Germany \\ ${ }^{3}$ Institute for Theoretical Physics, Utrecht University, Princetonpln 5, 3584 CC Utrecht, The Netherlands \\ ${ }^{4}$ H.H. Wills Physics Laboratory, University of Bristol, Tyndall Avenue, Bristol BS8 1TL, United Kingdom
}

\begin{abstract}
Quantitative predictions are presented of a depletion-induced torque and force acting on a single colloidal hard rod immersed in a solvent of hard spheres close to a planar hard wall. This torque and force, which are entirely of entropic origin, may play an important role for the key-lock principle, where a biological macromolecule (the key) is only functional in a particular orientation with respect to a cavity (the lock).

82.70.Dd,61.20.Gy,61.20.Ja
\end{abstract}

The depletion effect, i.e., the effect that smaller particles in a colloidal mixture are expelled from those regions where bigger particles are sufficiently close together, leads to an effective force between the bigger particles. In the case of hard-core interactions these effective forces are purely entropic in origin [1].2]. For the relatively simple geometries of two large spheres or a sphere close to a planar or curved wall in a solvent of small hard spheres this depletion force has been studied in detail in theory [3 [5], simulation [6.7], and experiment [8.9]. Depletion forces are crucial for the phase behavior of colloidal system; they can drive gas-liquid and liquid-solid phase separation, e.g., in colloid-polymer 110] and colloid-colloid mixtures [11]. It has been suggested [12 that depletion effects play an important role in the biological "key-lock" mechanism, in which a nonspherical macromolecule (the key) fits into a cavity (the lock) and forms a chemical bond, but only if the key has a particular orientation. A robust performance of this mechanism is possible if the biological environment is capable of passively transporting the key, correctly oriented, to the lock. This requires a chemically unspecific force to transport the center-of-mass of the macromolecule towards the cavity and a torque to orient it. In order to be able to study the relevance of the entropic contribution to this mechanism a quantitatively reliable tool for predicting such an entropic torque is needed. Using density functional theory and computer simulations, we show and predict quantitatively that depletion effects can generate both a force and a torque on a nonspherical particle in the vicinity of a planar wall thereby providing further evidence for the relevance of depletion phenomena in key-lock mechanisms.

The specific system we study is illustrated in Fig.1 and consists of (i) an infinite planar hard wall located at $z=0$, (ii) a hard spherocylinder with cylinder length $L$, diameter $\sigma$, at distance $z$ from the wall and forming an angle $\theta \in[0, \pi / 2]$ with respect to the wall normal, and (iii) a solvent of hard spheres with diameter $\sigma_{s}$ and number density $\rho_{s}$ in the bulk, i.e., far away from the wall. Since we are considering only hard interactions the temperature $T$ of the system plays only the role of an energy scale via $\beta=1 / k_{B} T$. We are interested in the effective rod-wall potential $W(z, \theta)$ induced by the spheres. The force and the torque on the rod follow by differentiating $-W(z, \theta)$ with respect to $z$ and $\theta$, respectively. The bare rod-wall interaction restricts the center of mass of the rod to $z$ to $z \geq z_{\min }(\theta)=$ $(\sigma+L|\cos \theta|) / 2$. The system, and hence $\beta W(z, \theta)$, is completely characterized by the aspect ratio $L / \sigma$ of the rod, the diameter ratio $\sigma / \sigma_{s}$, and the packing fraction $\eta=\rho_{s} \pi \sigma_{s}^{3} / 6$.

Following Asakura and Oosawa [1], a first approximation of the effective potential $W(z, \theta)$ can be obtained from the analysis of the volume that is excluded to the centers of the solvent spheres by the wall and the rod. The wall excludes the slab $0<z<\sigma_{s} / 2$, and the rod the volume of a spherocylinder of length $L$ and diameter $\sigma+\sigma_{s}$. If these two contributions to the excluded volume overlap, i.e., for $z<z_{\min }+\sigma_{s}$ (see Fig. 1) the volume accessible to the centers of the spheres and hence the entropy of the solvent increases. This gain of entropy translates directly into an effective, purely attractive force acting on the center of the rod. An important ingredient of this so-called Asakura-Oosawa approximation (AOa) is that the density profile of the spheres is taken to be constant and equal to the bulk density $\rho_{s}$, i.e., all correlations in the solvent are entirely neglected. The AOa therefore underestimates the strength and the range of the force acting on the rod.

Here we go beyond the AOa taking the sphere-sphere correlations into account. We recall that the depletion potential equals the grand-potential change of the (inhomogeneous) fluid of spheres in contact with the wall upon moving the rod from $z \rightarrow \infty$ to a finite value of $z$, at a given $\theta$. Within density functional theory (DFT), with

* present address: Max-Planck-Institut für Polymerforschung, Achermannweg 10, D-55128 Mainz, Germany 
$\mathcal{F}_{\text {ex }}\left[\rho_{s}, \rho_{r}\right]$ the excess (over ideal) free energy functional of a mixture of hard spheres and rods, one can write [5]

$$
\beta W(z, \theta)=\lim _{\rho_{r} \rightarrow 0}\left[c_{r}^{(1)}(z \rightarrow \infty, \theta)-c_{r}^{(1)}(z, \theta)\right],
$$

where $c_{r}^{(1)}(z, \theta)=-\delta \beta \mathcal{F}_{\text {ex }}\left[\rho_{s}, \rho_{r}\right] / \delta \rho_{r}(z, \theta)$ is the direct one-body correlation function of the rods [13] where $\rho_{r}(z, \theta)$ is the number density of rods for a given orientation $\theta$. The functional we use is based on Rosenfeld's fundamental measure theory (FMT) for mixtures of general convex hard bodies 14]. FMT has proved to account accurately for both the structure and the thermodynamics of inhomogeneous hard-sphere mixtures [15] as compared with simulations. Recently, a FMT for a mixture of spheres and needles of vanishing thickness was proposed [16]. Our extension to include a nonspherical particle with finite volume (the rod) in the theory requires the deconvolution of the Mayer- $f$ function of the rod-sphere interaction into a set of orientation dependent weight functions [14]. The details of this technically involved deconvolution will be explained elsewhere 17].

We obtain the depletion potential in two steps. First we calculate the unperturbed density profile $\rho_{s}(z)$ of the spheres close to the wall. This is the equilibrium profile in the limit $\rho_{r} \rightarrow 0$, as required in Eq.(1). The functional reduces, in this case, to the well-tested FMT for spheres. In the second step we use $\rho_{s}(z)$ to evaluate $c_{r}^{(1)}(z, \theta)$, using the deconvoluted Mayer- $f$ function. From Eq.(1D) we then obtain the depletion potential $W(z, \theta)$ for all $z$ and $\theta$ (from the profile $\rho_{s}(z)$ alone!).

We present results for $L / \sigma=10, \sigma / \sigma_{s}=1$, and a packing fraction $\eta_{s}=(\pi / 6) \sigma_{s}^{3} \rho_{s}=0.2239$ for the spheres. Figure 2 displays the depletion potential $\beta W(z, \theta)$ as function of $z$ and $\theta$. The first observation is that the potential is, unlike the AOa, not monotonic; the hard-sphere correlations generate a sequence of potential barriers and wells. The dashed and dotted curves in Fig. 目 denote the positions of the minima and maxima of the potential. We note that for small angles $\theta \ll 1$ the shape of the rod-wall depletion potential $W(z, \theta)$ coincides almost perfectly with the depletion potential $W_{w s}(z)$ between a sphere of diameter $\sigma$ and the wall, i.e., $W(z, \theta \ll 1) \approx W_{w s}(z-L \cos |\theta| / 2)$. For these small angles the length of the rod $L$ is rather unimportant for details of the depletion potential, as we have verified for various values of $L / \sigma$ and $\sigma / \sigma_{s}$. For large angles, however, the whole geometry of the rod, i.e., $L / \sigma$ and $\sigma / \sigma_{s}$, is relevant, and the depth of the depletion potential at contact becomes more negative as both $L / \sigma$ and $\sigma / \sigma_{s}$ are increased. In addition the contact value of the depletion potential can be further decreased by increasing $\eta_{s}$.

Although certain general trends of the influence of the geometry on the shape of the depletion potential can already be roughly understood within the simple AOa [1], the correlations in the hard-sphere fluid are very important and can lead to a quantitatively and even qualitatively different behavior. In Fig. 3 we illustrate this by comparing the depletion potential at contact as obtained within our DFT approach $\left(W\left(z_{\min }(\theta), \theta\right)\right.$, full line) with the corresponding quantity within the $\mathrm{AOa}\left(W^{A O a}\left(z_{\min }(\theta), \theta\right)\right.$, dotted line). While for small and large angles the contact value of the full depletion potential is more negative than within the AOa, for intermediate values of $\theta$ packing effects can shift the actual contact value above its corresponding AOa-value. If the rod is almost parallel to the wall, i.e., for $\theta \lesssim \pi / 2$ the deviation of the contact value calculated within the AOa from the prediction of the full DFT is significant and much more pronounced than in the well-studied wall-sphere or sphere-sphere geometry. The inset of Fig. 3 shows that a rod in contact with the wall can exhibit a metastable orientation for a certain angle $0<\theta_{0}<\pi / 2$ while the AOa predicts that this configuration is unstable. Although for the parameters under consideration here the height of the potential barrier is rather small $\left(0.007 k_{B} T\right)$, we note that its value increases for smaller values of $\sigma / \sigma_{s}$.

As a consequence of the dependence of the rod-wall depletion potential on the orientation $\theta$ for a given (fixed) distance $z$ of the center of the rod from the wall an entropic torque acts on the rod which drives it into an orientation with minimal depletion potential. This torque can be estimated by replacing the spherocylinder by a dumbbell composed of two spheres with diameter $\sigma$ connected by an infinitesimally thin but rigid wire of length $L$. Since the sphere-wall depletion force $f_{s w}(z)$ acts on the two spheres located at $z_{ \pm}=z \pm(L / 2) \cos \theta$ the corresponding torque with respect to the center of mass is given by

$$
M_{d b}(z, \theta)=\frac{L}{2} \sin \theta\left[f_{s w}\left(z_{-}\right)-f_{s w}\left(z_{+}\right)\right] .
$$

It turns out that at low packing fractions of the hard-sphere solvent, or at sufficiently large separations of the rod from the wall Eq. (2) yields a semi-quantitative expression for the torque acting on a spherocylinder.

From the full rod-wall depletion potential $W(z, \theta)$ one can obtain the torque by rotating the rod by an infinitesimal angle $d \theta$ around an axis through the center of the rod in a direction characterized by the unit vector $\mathbf{n}_{\theta}$ normal to the symmetry plane shown in Fig. 1. The corresponding change in the depletion potential is $d W(z, \theta)$ which can be written as $d W=-\sum_{i} \mathbf{f}_{i} \cdot d \mathbf{r}_{i}$, i.e., as a sum of forces $\mathbf{f}_{i}$ acting on the rod at positions $\mathbf{r}_{i}$ from the center with $d \mathbf{r}_{i}=d \theta\left(\mathbf{r}_{i} \times \mathbf{n}_{\theta}\right)$. It follows that the torque $\mathbf{M}(z, \theta)=M(z, \theta) \mathbf{n}_{\theta}$ with respect to the center of mass is given by 


$$
M(z, \theta)=-\frac{\partial W(z, \theta)}{\partial \theta} .
$$

The symmetry of the problem leads to $M(z, \theta)=0$ for $\theta=0$ and $\theta=\pi / 2$. A positive value of the torque acts on the rod as to increase the angle $\theta$ (rotating it parallel to the wall) while a negative value of $M$ leads to a decrease of $\theta$ (rotating it normal to the wall). Some typical examples for the torque as a function of $\theta$ for various values of $z$ are shown in Fig. 1 . Lines and symbols denote results from DFT and molecular dynamics (MD) simulations, respectively. The DFT predictions are in excellent agreement with the simulations. The details of the simulations will be presented elsewhere [17].

For larger distances of the rod from the wall $W(z, \theta)$ exhibits local minima and maxima in addition to the global minimum with corresponding zeros of the torque (see Fig. 6 for $z=5.5 \sigma_{s}$ and $z=4.5 \sigma_{s}$ ). A minimum (maximum) of $W(z, \theta)$ leads to a zero of $M(z, \theta)$ with a negative (positive) gradient in $\theta$. Furthermore, the torque can exhibit a cusp (see Fig. (1) if the minimal distance between the wall and the rod leaves space for precisely one solvent sphere. This can be realized for distances $\sigma_{s}+\sigma / 2 \leq z \leq \sigma_{s}+(L+\sigma) / 2$ and a corresponding orientation $\theta_{\text {cusp }}=\arccos \left(\left(2\left(z-\sigma_{s}\right)-\sigma\right) / L\right)$.

As the rod moves closer to the wall the modulus of the torque increases and for small separations from the wall (see, e.g., $z=1.5 \sigma_{s}$ in Fig. (1) the torque vanishes only for $\theta=\pi / 2$ which is, however, only a metastable configuration. For such small and fixed values of $z$ the entropic torque rotates the rod towards configurations with smaller angles until the rod is in contact with the wall and has to stop its rotation. Thus the rod reaches its most favorable configuration of lying parallel in contact with the wall not by approaching the wall in a parallel configuration but by touching the wall first at one end and then by decreasing the distance of its center from the wall. The modulus of the maximum of the entropic torque for the system considered here is of the order of about $20 k_{B} T \mathrm{rad}^{-1}$, which corresponds to roughly $10^{-20} \mathrm{~J} \mathrm{rad}^{-1}$ at room temperature. The strength of the torque increases for larger values of $L / \sigma, \sigma / \sigma_{s}$, or $\eta_{s}$. For example, for $L / \sigma=20, \sigma / \sigma_{s}=2$, and $\eta_{s}=0.2239$, the modulus of the maximum of the entropic torque reaches a value of about $38.7 k_{B} T \mathrm{rad}^{-1}$. We note that the maximum torque acts at small values of $z$ for which the AOa also would predict the existence of a torque; however, its magnitude is largely enhanced through the correlations in the solvent.

With the results presented so far we can comment on some aspects of the path in the $z-\theta$ plane a rod would take upon approaching the wall from the bulk. Only if the rod comes sufficiently close to the wall to be subject to the oscillations of the number density of the hard-sphere fluid as a function of $z$, the entropic force and torque will act on it. The closer the rod gets to the wall the higher are the potential barriers between the minima (see Fig. 2) the rod has to overcome by thermal motion in order to move still closer. The potential barriers are, however, only moderate and easy to overcome for small angles and increase for angles $\theta \gtrsim 60$ degrees so that it is not optimal to rotate the rod until it is parallel to the wall since then the potential barrier for further approach is largest and for the parameters considered here more than $2.3 k_{B} T$. On the other hand along the line of the second minimum - see the dashed lines in Fig. 2- $\beta W$ has a similar shape as $\beta W\left(z_{\min }(\theta), \theta\right)$ [see Fig. 3], so that the entropic torque will rotate the rod into the local minimum at $\theta=\pi / 2$ if $\theta$ is already sufficiently large. Once the rod is in contact with the wall the torque will rotate it towards the pronounced global minimum of $\beta W(z, \theta)$.

We have presented quantitative predictions of the entropic torque acting on a hard spherocylinder close to a hard planar wall in a solvent of small hard spheres. Our DFT predictions are in excellent agreement with our MD simulations. We find that the the depletion effect leads to a significant entropic torque and it is tempting to speculate that this entropic torque can play an important role in understanding the key-lock principle.

It is a pleasure to thank R. Evans, M. Schmidt, C. Bechinger, and M. P. Allen for stimulating discussions. This work is part of the Research program of the "Stichting voor Fundamenteel Onderzoek der Materie (FOM)", which is financially supported by the "Nederlandse Organisatie voor Wetenschappelijk Onderzoek (NWO)". D.A. acknowledges the support of the Overseas Research Students Grant, EPSRC grants GR/L89990, GR/M16023, INTAS grant 9900312. MD simulations used the GBMEGA program of the 'Complex Fluids Consortium' with computer time allocated at the CSAR facility.

[1] S. Asakura and F. Oosawa, J. Chem. Phys. 22, 1255 (1954); J. Polymer Sci. 33, 183 (1958).

[2] A. Vrij, Pure and Appl. Chem. 48, 471 (1976).

[3] R. Roth, B. Götzelmann, and S. Dietrich, Phys. Rev. Lett. 83, 448 (1999). 
[4] B. Götzelmann, R. Roth, S. Dietrich, M. Dijkstra, and R. Evans, Europhys. Lett. 47, 398 (1999); R. Roth, doctoral thesis, Bergische Universität Wuppertal (1999).

[5] R. Roth, R. Evans, and S. Dietrich, Phys. Rev. E 62, 5360 (2000).

[6] T. Biben, P. Bladon, and D. Frenkel, J. Phys.: Condens. Matter 8, 10799 (1996).

[7] R. Dickman, P. Attard, and V. Simonian, J. Chem. Phys. 107, 205 (1997).

[8] J.C. Crocker, J.A. Matteo, A.D. Dinsmore, and A.G. Yodh, Phys. Rev. Lett. 82, 4352 (1999).

[9] D. Rudhardt, C. Bechinger, and P. Leiderer, Phys. Rev. Lett. 81, 1330 (1998).

[10] M. Dijkstra, J.M. Brader, and R. Evans, J. Phys.: Condens. Matter 11, 10079 (1999).

[11] M. Dijkstra, R. van Roij, and R. Evans, Phys. Rev. Lett. 81, 2268 (1998); ibid 82, 117 (1999); Phys. Rev. E 59, 5744 (1999).

[12] see, e.g., M. Kinoshita and T. Oguni, Chem. Phys. Lett. 351, 79 (2002) and references therein.

[13] R. Evans, Adv. Phys. 28, 143 (1979).

[14] Y. Rosenfeld, Phys. Rev. E 50, R3318 (1994); Mol. Phys. 86, 637 (1995).

[15] Y. Rosenfeld, Phys. Rev. Lett. 63, 980 (1989); J. Chem. Phys. 98, 8126 (1993).

[16] M. Schmidt, Phys. Rev. E 63, 050201-1 (2001).

[17] R. Roth, R. van Roij, D. Andrienko, K. R. Mecke, and S. Dietrich, unpublished. 


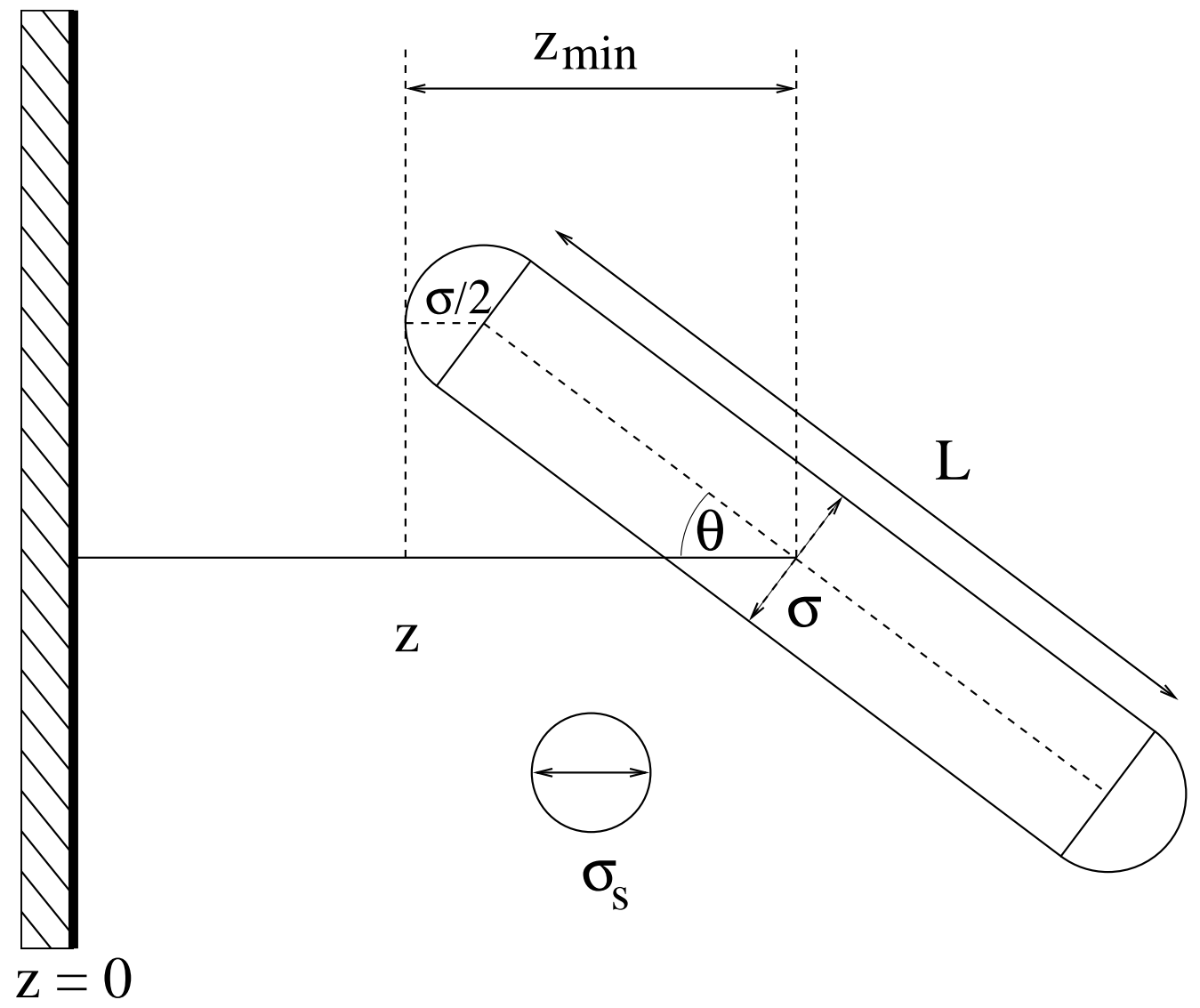

FIG. 1. A spherocylinder of length $L$ and diameter $\sigma$ at angle $\theta$ and distance $z$ relative to a a planar hard wall at $z=0$. The minimal value of $z$ is $z_{\min }(\theta)=(\sigma+L|\cos \theta|) / 2$. The $\operatorname{rod}$ is immersed in a solvent of hard spheres of diameter $\sigma_{s}$ and number density $\rho_{s}$ far from the wall. 


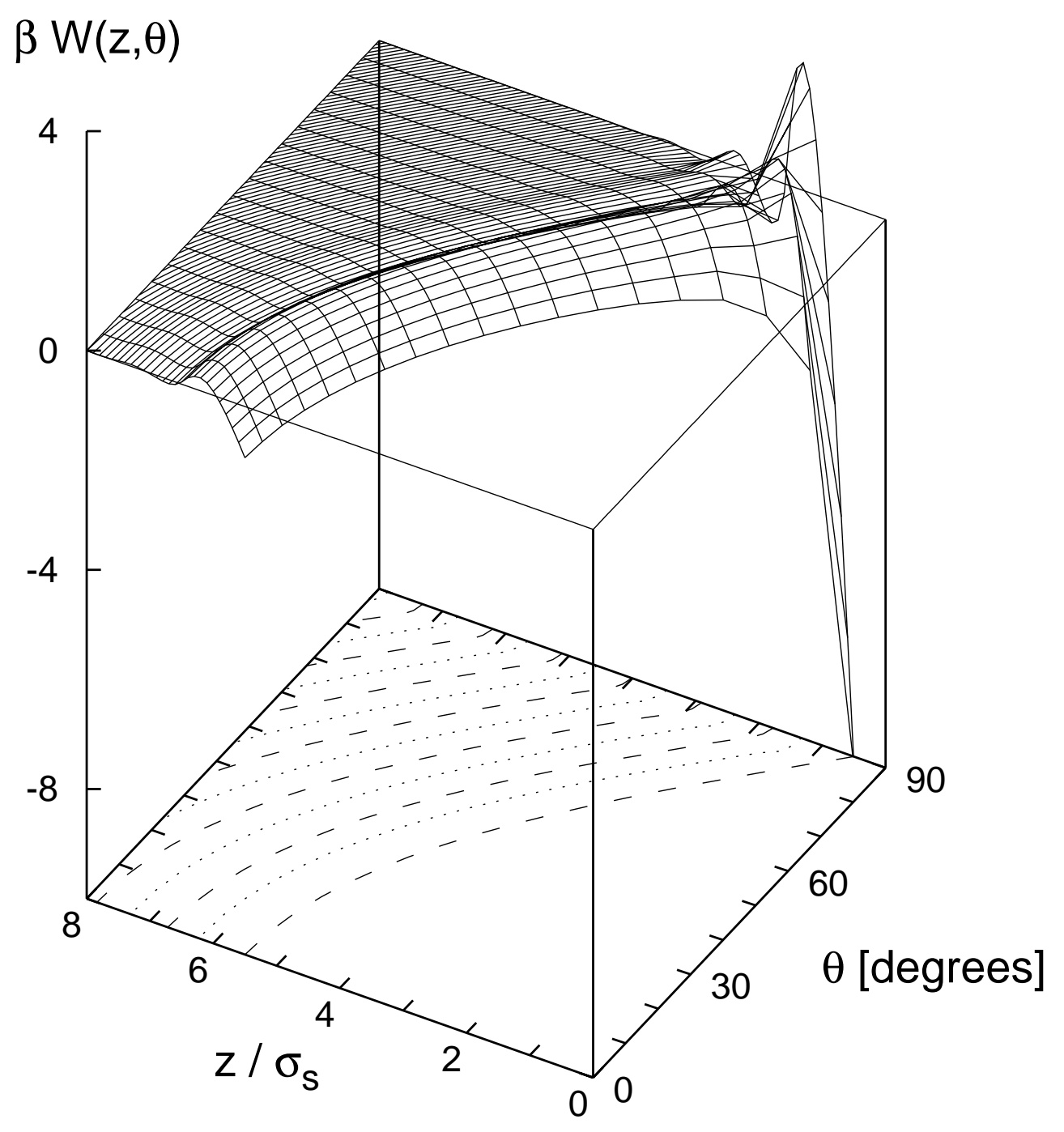

FIG. 2. Depletion potential $\beta W(z, \theta)$ for the spherocylinder shown in Fig. 目 for $L / \sigma=10, \sigma / \sigma_{s}=1$, and a packing fraction $\eta_{s}=\rho_{s} \pi \sigma_{s}^{3} / 6=0.2239$ of the hard-sphere solvent. $\theta$ is measured in degrees. The dashed and dotted lines represent the positions of local minima and maxima, respectively. 


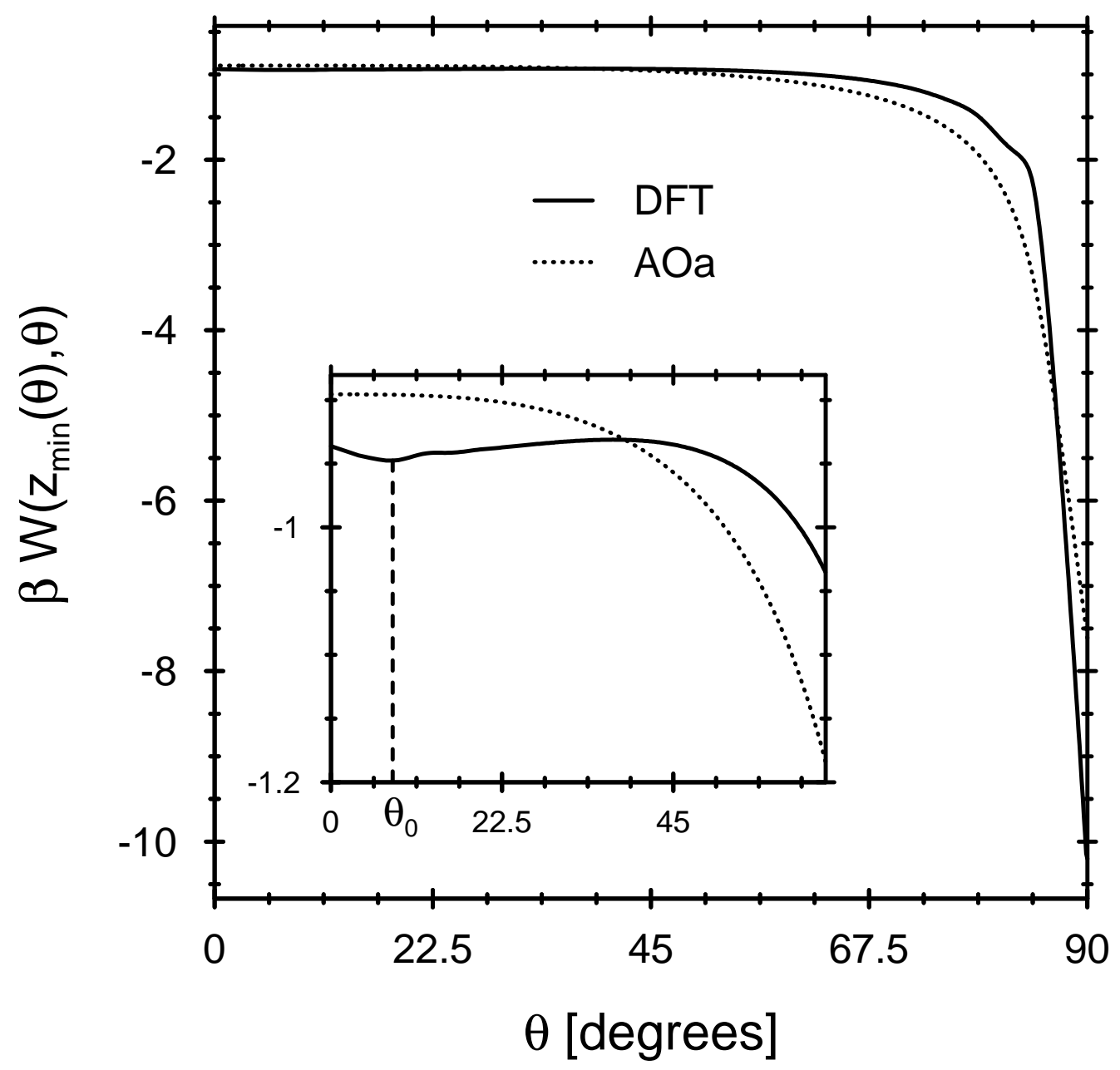

FIG. 3. The contact value $\beta W\left(z_{\min }(\theta), \theta\right)$ as a function of $\theta$ (in degrees) as calculated within DFT (full line) and within the AOa (dotted line) for the system described in Fig. 2. The inset shows that a rod in contact with the wall can exhibit a metastable orientation for $\theta=\theta_{0} \approx 8$ degrees with a barrier height $\beta \Delta W=0.007$. 


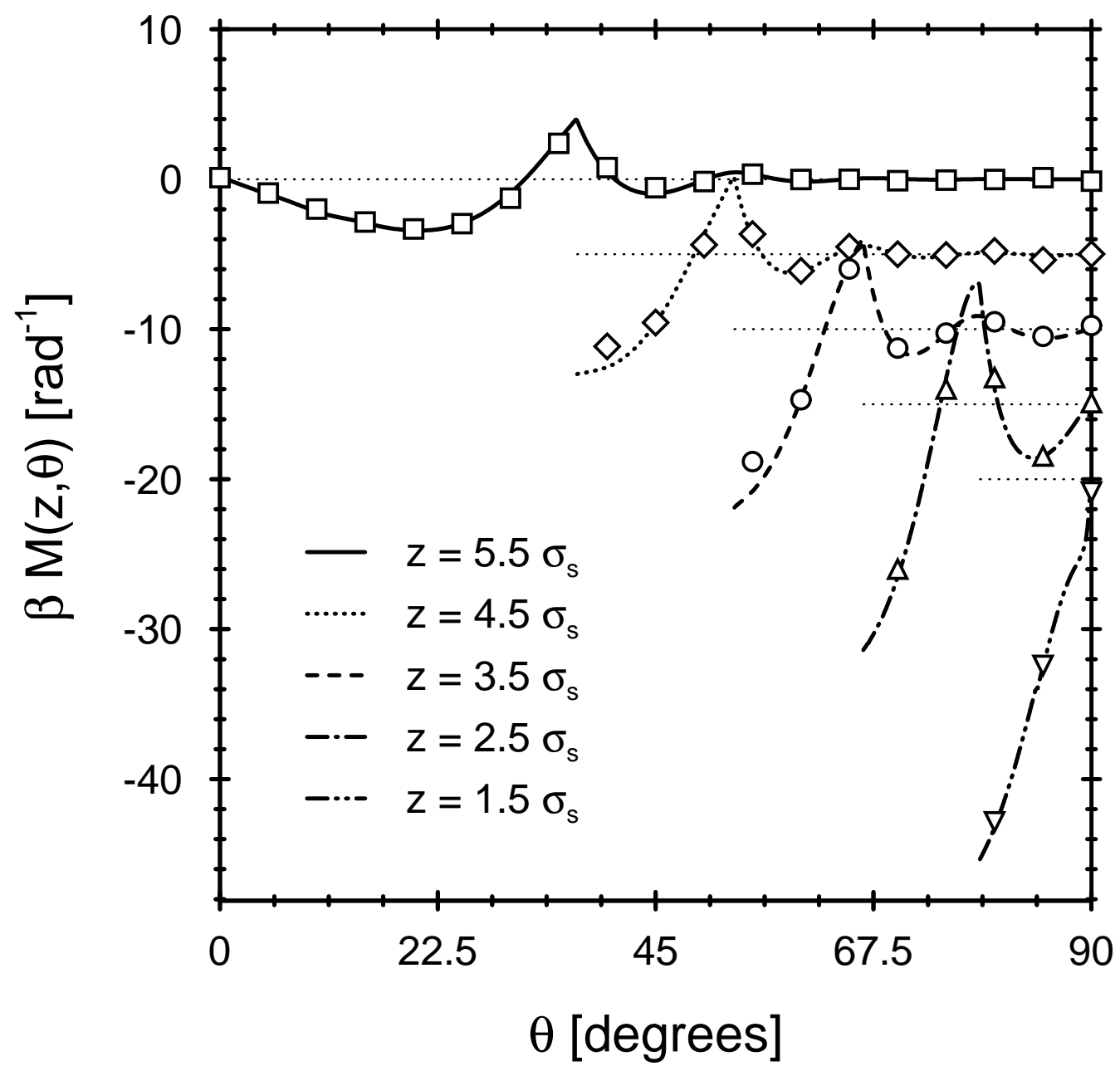

FIG. 4. The torque $\beta M(z, \theta)$ as function of $\theta$ for various values of $z$. For reasons of clarity the curves for $z=4.5,3.4, \ldots$ are shifted downward by $-5,-10, \ldots$, respectively. The horizontal lines $M=0$ are indicated partially. The parameters of the system are the same as in Fig. 2. Because of the hard wall at $z=0$ the torque is defined only for $\theta \geq \arccos (2 z / L-\sigma / L)$ if $z<(\sigma+L) / 2 . M>0(<0)$ corresponds to a force which tends to align the rod parallel (normal) to the wall. The lines denote our DFT results which are in excellent agreement with our simulations (symbols). The error bars of the simulations are of the order of the symbol size. 